\title{
THE TWENTY-EIGHTH ANNUAL MEETING OF THE AMERICAN MATHEMATICAL SOCIETY
}

The twenty-eighth annual meeting of the Society and the forty-eighth regular meeting of the Chicago Section were held at the University of Toronto on Wednesday and Thursday, December 28-29, 1921, in affiliation with the Convocation week meetings of the American Association for the Advancement of Science. Dormitory and eating accommodations for the mathematical group were furnished by the University of Toronto, Knox College being almost wholly given up to this purpose. Hart House was the center of a generous hospitality which filled the hours outside the scientific program with a delightful and varied entertainment.

On Thursday morning there was a joint session with Sections $\mathrm{B}$ and $\mathrm{C}$ of the American Association for the Advancement of Science and with the American Physical Society, and on Thursday afternoon a joint session with Section A and the Mathematical Association of America. At a special Convocation, the University of Toronto conferred on Professor E. H. Moore, as president of the American Association for the Advancement of Science, the degree of Doctor of Science, honoris causa.

The attendance included the following eighty-three members of the Society: Archibald, G. N. Armstrong, Atchison, Bacon, Beatty, Beetle, Bliss, Bradshaw, R. W. Burgess, Cairns, H. K. Cummings, L. D. Cummings, Currier, Curtiss, Dadourian, DeLury, Denton, L. L. Dines, Dostal, Dresden, Peter Field, Fields, Findlay, Finkel, Focke, W. B. Ford, J. L. Gibson, Gillespie, Glashan, Glenn, Glover, Gummer, Haskins, Hazlett, E. R. Hedrick, Howe, Huntington, Hurwitz, Ingraham, Karpinski, Kingston, Lennes, F. P. Lewis, Marshall, T. E. Mason, G. A. Miller, Norman Miller, Mirick, E. H. Moore, F. R. Moulton, Newkirk, Olson, F. W. Owens, H. B. Owens, Leigh Page, Pell, Phillips, Pitcher, Pounder, A. V. 
Richardson, R. G. D. Richardson, H. L. Rietz, Robison, E. D. Roe, Roever, Sheppard, Sinclair, Slaught, C. E. Smith, Edwin R. Smith, P. F. Smith, Synge, Uhler, Veblen, Waddell, Watkeys, Webster, Wiener, Wilder, W. L. G. Williams, A. H. Wilson, E. B. Wilson, J. M. Young.

President Bliss occupied the chair at the regular meetings on Wednesday, being relieved at the morning session by Professor P. F. Smith and at the afternoon session by Professor C. N. Haskins. The joint session of Thursday morning was presided over by Professors G. W. Stewart and W. D. Harkins, Vice-Presidents of Sections B and C respectively, and that of Thursday afternoon by Professor Oswald Veblen, Vice-President of Section A.

The Council announced the election of the following sixtytwo persons to membership in the Society:

Professor William Cyrus Bartol, Bucknell University; Professor William John Bauduit, Howard University;

Mr. Benjamin Berman, University of Maryland;

Miss Florence Lucile Black, University of Kansas;

Professor Edmond Wesley Bowler, New Hampshire College;

Professor John Wentworth Clawson, Ursinus College;

Professor Robert Cameron Colwell, Geneva College;

Professor Charles Winthrop Crockett, Rensselaer Polytechnic Institute;

Mr. Herbert Kimball Cummings, Brown University;

Professor Lloyd Slote Dancey, Carroll College;

Mr. William Whitfield Elliott, Cornell University;

Professor John Thomas Erwin, George Washington University;

Professor Earl Frederick Farnau, University of Cincinnati;

Professor Forest Almos Foraker, University of Pittsburgh;

Mr. William McKinley Gafafer, Columbia University;

Mr. Harry Merrill Gehman, University of Pennsylvania;

Professor Albert Henry Steward Gillson, McGill University;

Professor J. W. Harrell, Baylor University;

Professor George Abram Harter, University of Delaware;

Miss Eugenie Caroline Hausle, Columbia University;

Professor Ruby Usher Hightower, Shorter College;

Mr. Van Buren Hinsch, Missouri School of Mines;

Mr. Frank Adin Joy, New Hampshire College;

Professor Oscar Godfrey Lawless, Talladega College;

Mr. Maurice Moses Levita, Temple University;

Mr. Thomas Richard Long, University of Rochester;

Professor Frederick Milton McGaw, Cornell College;

Professor James McGiffert, Rensselaer Polytechnic Institute; 
Mrs. Martha (Macdonald) McKelvey, Iowa State College;

Professor Neil Bruce MacLean, University of Manitoba;

Miss Ida Caroline Millay, New Hampshire College;

Professor Clifford Newton Mills, Heidelberg University;

Professor Eugenie Maria Morenus, Sweet Briar College;

Dr. Hermance Mullemeister, University of Washington;

Dean Albert O'Brien, O. F. M., St. Bonaventure's College;

Mr. Frederick Allen Parker, West Virginia Collegiate Institute;

Professor Annie McKinnie Pegram, Greensboro College;

Mr. Cecil Glenn Phipps, University of Minnesota;

Professor Joseph Romeo Plante, Viator College;

Mr. Louis Gordon Pooler, Columbia University;

Mr. Abraham Press, Patent Section, Air Service, War Department;

Professor George Edwin Ramsdell, Bates College;

Dean Otis Everett Randall, Brown University;

Professor Joseph Marshall Rankin, College of Idaho;

Mr. George Emil Raynor, Princeton University;

Professor Arthur Vernon Richardson, Bishop's College;

Professor Tom Gladstone Rodgers, New Mexico Normal University;

Professor Sidney Archie Rowland, Jr., Union College;

Professor Henry Alford Ruger, Columbia University;

Professor Joseph Seidlin, Alfred University;

Mr. Leonard Shaffer, New Hampshire College;

Professor Rowland Alfred Sheets, Denison University;

Professor Wilfred H. Shirk, University of Buffalo;

Mr. George Yale Sosnow, Prudential Insurance Company;

Professor Guy Greene Speeker, Michigan Agricultural College;

Professor Mary Cass Spencer, Newcomb College, Tulane University;

Mr. Joe Ichino Tanoue, Columbia University;

Mr. Louis Weisner, Columbia University;

Professor John Jefferson Wheeler, University of Kansas;

Mr. Daniel Everett Whitford, University of Rochester;

Professor Hugh Carey Willett, University of Southern California;

Miss Ruby Willis, Wellesley College.

The invitation extended by the University of Rochester to hold the summer meeting of 1922 at that institution was accepted, and Professors Gale (chairman), Hildebrandt, Metzler, Watkeys, and the Secretary were constituted a committee on arrangements. Professor O. D. Kellogg and the Secretary were appointed as representatives of the Society on the Council of the American Association for the Advancement of Science for 1922 .

The Council voted to request that the space occupied in the Bulletin by an abstract of a paper presented to the Society 
should be limited to the equivalent of two hundred words of text, and that display typography be avoided.

The total membership of the Society is now 1005, including 85 life members. The total attendance of members at all meetings, including sectional meetings, during the past year was 420 ; the number of papers read was 175 . The number of members attending at least one meeting was 273 . At the annual election 169 votes were cast.

The reports of the Treasurer and of the Auditors (Mr. S. A. Joffe and Professor P. H. Linehan) were received, showing a balance of $\$ 10,604.22$, including the Life Membership Fund, which now amounts to $\$ 7,528.87$. Sales of the Society's publications during the year amounted to $\$ 3,222.16$.

The Library now contains 6,014 volumes, excluding some 500 unbound dissertations, now being catalogued.

At the annual election, which closed on Wednesday morning, the following officers and other members of the Council were chosen:

Vice-Presidents, Professor R. D. Carmichael, Professor D. E. SMith.

Secretary, $\quad$ Professor R. G. D. Richardson.

Treasurer, $\quad$ Professor W. B. Fite.

Librarian, Professor R. C. ARChIBALD.

\section{Committee of Publication}

Professors E. R. Hedrick, W. A. Hurwitz, J. W. Young.

Members of the Council to serve until December, 1924

Professor J. W. Alexander, Professor L. L. Dines, Professor Henry Blumberg, Professor F. R. Sharpe.

At the end of the afternoon session of Wednesday, there was a business meeting of the Chicago Section. The officers elected for the next biennium are Professor A. B. Coble, Chairman; Professor Arnold Dresden, Secretary; and Professor E. W. Chittenden. 
The committee on the symposium for April, 1922, reported that the symposium lecture would be given by Professor A. B. Coble on Cremona transformations and applications to algebra, geometry, and modular functions.

The meeting of the Society immediately preceded that of the Mathematical Association of America. A very pleasant occasion was the joint dinner, on Friday evening, of the various mathematical, physical, and astronomical associations. At the afternoon session of Wednesday it was voted to express the thanks of the Society for the hospitality of the Royal Canadian Institution and the University of Toronto, and for the entertainment furnished by the local committee of the American Association for the Advancement of Science.

On Thursday morning the program was as follows:

I. Atomic nuclei and extra-nuclear electronic configuration, by Professor J. C. McLennan, retiring Vice-President of Section B.

II. Symposium on quantum theory: for Section C, Dr. R. C. Tolman; for the American Mathematical Society, Professor H. B. Phillips; for the American Physical Society, Dr. Saul Dushman.

At the joint session on Thursday afternoon, the following addresses were given:

I. A mechanical analogy in the theory of equations, by Professor D. R. Curtiss, retiring Vice-President of Section A.

II. The research information service of the National Research Council, by Professor R. M. Yerkes, of the National Research Council.

III. Subsidy funds for mathematical projects, by Professor H. E. Slaught.

IV. Algebraic guides to transcendental problems, by Professor R. D. Carmichael, retiring Chairman of the Chicago Section.

In the absence of Professor Carmichael, an abstract of his paper was given by Professor Dresden; the paper appears in full in this number of the Bulletin. Professor Slaught's paper has appeared in SCIENCE. 
Titles and abstracts of the papers read at the regular sessions on Wednesday follow below. Professor Schwatt's first paper was read by Professor Pounder. The papers of Dr. Zeldin, Professors Crum, Forsyth, and Emch, Dr. Hille, Professors Lipka, MacNeish, Everett, Dodd, Wilczynski, and Glenn, Professor Schwatt's second paper and that of Dr. Wiener and Dr. Walsh were read by title.

1. Professor C. E. Wilder: Differential geometry of an mdimensional manifold in a euclidean space of $n$ dimensions.

The differential geometry of $(n-1)$-dimensional varieties in an $n$-dimensional space has long been developed. More recently several authors have treated the two-dimensional manifold in $n$ dimensions.

In this paper Professor Wilder takes up the theory of the intermediate cases and generalizes certain theorems and methods of ordinary surface theory. The method used is that of the absolute calculus of Ricci combined with $n$-dimensional vector analysis.

2. Professor C. E. Wilder: Differential geometry of an mdimensional manifold in a euclidean space of $n$ dimensions. Second paper.

The $m$-dimensional manifold in $n$ dimensions not only has extension in different directions as has the surface in ordinary space, but also it winds its tortuous way through $n$ dimensions as does the twisted curve in three dimensions. In this paper certain facts of curve theory are generalized to an $m$-dimensional manifold in $n$ dimensions.

3. Mr. M. H. Ingraham: A modification of Peano's postulates for positive integers.

In this paper is studied the complete independence of Peano's postulates for positive integers, and of Padoa's modification of these postulates. This study is made by means of their complete existential theory, as introduced by E. H. Moore in his New Haven Colloquium Lectures, 1906. It is proved that these postulates are not completely independent. A modification of these systems of postulates is given which is completely independent. 
4. Professors L. P. Eisenhart and Oswald Veblen: The Riemann geometry and its generalizations.

The authors determine the conditions under which the $n$ dimensional geometry of a system of paths defined by the solution of a system of differential equations

$$
\frac{d^{2} x^{i}}{d s^{2}}=\Gamma_{j k}{ }^{i} \frac{d x^{j}}{d s} \frac{d x^{k}}{d s}
$$

in which the $\Gamma$ 's are functions of $x^{1}, x^{2}, \cdots, x^{n}$ subject only to the condition $\Gamma_{j k}^{i}=\Gamma_{k j}^{i}$ reduces to the Riemann geometry determined by a quadratic form

$$
d s^{2}=g_{i j} d x^{i} d x^{j} .
$$

The conditions obtained are such as to indicate that the metric introduced by Weyl as the basis of combined electromagnetic and gravitation theory is next to the Riemann metric in order of simplicity. The paper appears in the Proceedings of the National Academy, February, 1922.

5. Professor R. W. Burgess: The problem of apportionment. The method of the weighted geometric mean.

If $N$ representatives are to be apportioned among several states of total population $P$, the true quota for a state of population $A$ is $\alpha=A N / P$, ordinarily a fraction. The number of representatives actually assigned the state must be an integer, say $a$. The ratio $r=a / \alpha$ or $\alpha / a$, whichever is greater than unity, is fundamental in the consideration of the problem. Professor Burgess suggests a method of apportionment based on the following single postulate: That apportionment is most satisfactory for which the continued product of terms of the type $r^{A}$, one term for each state, is smallest. This in effect takes the ratio for each inhabitant of each state, and uses the product of all such ratios as the measure of unfairness. If the exponents of the $r$ were in each case unity, i.e., if the ratio were taken once for each state rather than once for each individual, this method would, in the usual case, become equivalent to those proposed by Dr. Hill and Professor Huntington. As in this new method the quantity $[\alpha / \sqrt{a(a+1)}]^{A}$ plays about the same rôle as does $A / \sqrt{a(a+1)}$ in Professor Huntington's method, and $A /(a+1 / 2)$ in Professor Willcox's method, it may be called the method of the weighted geometric mean. 
6. Professor E. V. Huntington: Necessary and sufficient conditions in the problem of apportionment.

The present paper compares the method of major fractions and the method of equal proportions by analyzing all the known conditions or tests (including several new ones) which have been suggested in favor of either of them.*

The real question is believed to be one of simple equality between each state and every other state, here and now; and the tests based on this idea point clearly to the method of equal proportions.

Tests which attempt to compare each state directly with a norm for the whole country are found to be, in general, indeterminate or unworkable. Tests based on the idea of a total or average deviation from the norm lead to so many different methods that a choice among them appears difficult to make, especially when the averaging process is extended over unlimited time.

7. Dr. S. D. Zeldin: Commutativity of contact transformations of mechanics.

It is well known that with every conservative dynamical system, defined by its potential energy (a function of the generalized coordinates) and its kinetic energy (a quadratic function of the generalized velocity components), an infinitesimal contact transformation is associated whose characteristic function is of a special type. In this paper Dr. Zeldin proves that if two contact transformations associated with dynamical systems, defined as above, are independent, they cannot be commutative.

8. Professor G. A. Miller: Substitutions commutative with every substitution of an intransitive group.

This paper appears in full in this number of the Bulletin.

* For full bibliography up to the middle of 1921 (with special reference to the work of J. A. Hill) see $A$ new method of apportionment, by E. V. Huntington, Quarterly Publication of the American Statistical Association, September, 1921. See also Report of the Advisory Committee to the Director of the Census, ibid., December, 1921, and a forthcoming paper by F. W. Owens in the same journal. Letters from E. V. Huntington and W. F. Willcox have appeared in the New York Times for Oct. 18, Oct. 23, Nov. 13, and Dec. 11, 1921. Recent papers have been presented to the American Mathematical Society by E. V. Huntington, Sept. 8, and R. W. Burgess, Dec. 28, 1921. 
9. Professor G. A. Miller: Seeming contradictions in the theory of groups.

There are at least three distinct definitions of the term "same group" in common use in the theory of groups of finite order: (1) two substitution groups are the same if and only if one can be transformed into the other by a substitution; (2) two substitution groups or two abstract groups are the same if and only if a simple isomorphism can be established between their substitutions or operators; (3) two groups are the same if and only if one is obtained from the other by an interchange of operators. Various seeming contradictions that arise from the use of these different definitions are pointed out.

10. Professor W. A. Hurwitz: Convergence-factors in Cesàrosummable series.

Sufficient conditions for the theorem on convergence-factors for Cesàro-summable series have been given by C. N. Moore, Hardy, Bromwich and Chapman. Results essentially identical with part of the theorem have been proved by Schur, Carmichael and others. By means of a slight further generalization of the Silverman-Toeplitz criterion as extended by Hildebrandt and Carmichael, the author establishes necessary and sufficient conditions for the convergence-factor theorem in the case of Cesàro-summability of any order, real or complex, with positive real component.

11. Dr. W. L. Crum: Note on the determination of the rectilinear secular trend of an ordered series of statistical relatives.

In the analysis of certain historical series, the question arises whether, in determining the position of the line by the method of least squares, the relative deviations should not be used rather than the absolute deviations. The paper determines the line, to a first approximation, by using relative deviations, and shows that the divergence from the line as ordinarily determined is not likely to be of importance for practical applications; but there are significant theoretical consequences.

12. Professor C. H. Forsyth: Provisions for depreciation based directly upon appraisal.

Practically all methods of computing the depreciation charge succeed in providing a fund which will accumulate and ultimately meet the expense of replacing the property at the end 
of its lifetime, but no definite relation is maintained between the amount in the fund and the total amount of depreciation at any intermediate date. A method of computing the annual depreciation charge is given wherein estimates of periodic depreciation by an efficient appraiser are the foundation and accumulations of interest upon the fund are employed to reduce later charges. The scheme then insures a constant equality between the true wearing value and the theoretical wearing value with a degree of accuracy which will depend upon the reliability of the appraiser's estimates.

13. Professor Arnold Emch: Plane algebraic curves invariant under a given quadratic Cremona transformation.

The transformation which the author chooses in this paper has the well known simple form $\rho x_{1}^{\prime}=x_{2} x_{3}, \rho x_{2}{ }^{\prime}=x_{3} x_{1}$, $\rho x_{3}{ }^{\prime}=x_{1} x_{2}$, with $A_{1}(1,0,0), A_{2}(0,1,0), A_{3}(0,0,1)$ as the base, and $B(1,1,1), B_{1}(-1,1,1), B_{2}(1,-1,1), B_{3}(1,1,-1)$ as the invariant points. The following theorem is proved by means of a net of invariant cubics: Let $C_{n}$ be an invariant $n$-ic under the given transformation with ordinary multiple points of possible orders $\mu_{1}, \mu_{2}, \mu_{3}, \nu, \nu_{1}, \nu_{2}, \nu_{3}$ at the $A$ 's and $B$ 's respectively. Then $\mu_{1}+\mu_{2}+\mu_{3}=n$, and the lines joining corresponding points of the transformation on the $C_{n}$ envelope a curve $\mathfrak{E}_{m}$ of class $m=n-1 / 2\left(\nu+\nu_{1}+\nu_{2}+\nu_{3}\right)$. Conversely, the locus of pairs of corresponding points on the tangents of any curve $\mathfrak{S}_{m}$ is a certain invariant $C_{n}$. All invariant $C_{n}$ 's may be generated in this manner. When the $\mathbb{E}_{m}$ is in a general position, then $C_{n}$ is of order $n=3 m$. In case of a rational $\mathbb{E}_{m}$, the $C_{n}$ is, in general, hyperelliptic.

As an application Professor Emch studies hyperelliptic sextics, and rational invariant curves.

14. Professor Joseph Lipka: Canonical systems and the general problem of dynamics.

In this paper, Professor Lipka first derives the most general infinitesimal homogeneous contact transformations in $2 n$ variables, $x_{1}, x_{2}, \cdots, x_{n}, p_{1}, p_{2}, \cdots, p_{n}$, connected by a relation $H\left(x_{1}, \cdots, x_{n}, p_{1}, \cdots, p_{n}\right)=$ constant, which leave the Pfaffian $\Sigma_{i} p_{i} d x_{i}$ invariant. Interpreting this result properly in a metric space defined by $d s^{2}=\Sigma_{i k} a_{i k} d x_{i} d x_{k}$, the equations of the transformation reduce to the canonical equations of the dynamical trajectories in a conservative field of force in a curved space of $n$ dimensions. We thus get a very elegant 
and very brief proof of the characteristic property of orthogonality of a system of dynamical trajectories (or of any natural family of curves)-including both the direct theorem of Lipschitz $^{*}$ and its converse as given by the writer. $\dagger$

\section{Professor H. F. MacNeish: Euler squares.}

Euler proposed the following problem called "the problem of the 36 officers": Six officers of different rank are chosen from each of six different regiments. It is required to arrange them in a solid square so that no two officers of the same rank or of the same regiment shall be in the same row or the same column. This is equivalent to the problem of arranging in a square array the 36 distinct two-digit numbers with digits $1,2,3, \cdots, 6$ so that there are no repetitions in the columns or in the rows either in the units' digits or in the tens' digits. This array is called an Euler square of order 6, degree 2 and index 6, 2. Euler stated without proof that the solution is impossible for order $n \equiv 2(\bmod 4)$. By associating the Euler square with a geometric configuration, the author gives a proof by analysis situs. Methods are given for constructing Euler squares by means of substitution groups and by combining those of lower order. Euler squares are applied to the problem of making schedules for tournaments.

16. Professor H. S. Everett: The expression of general forms as determinants whose elements are forms. Preliminary report.

The author obtains the following theorems: (1) If a general form of degree $\rho r$ in $n \geqq 2$ variables has more than $[n(n+1) \cdots(n+\rho-1) / \rho !-2] r^{2}+2$ terms, it cannot be expressed as a determinant of order $r \geqq 2$ whose elements are forms of degree $\rho \geqq 2$ in the $n$ variables. (2) A general form of degree $\rho r$ in $n \geqq 2$ variables cannot be so expressed if $n>3$.

These theorems are extensions of similar theorems in a paper by L. E. Dickson (Transactions of this Society, April, 1921), in which general forms expressible as a determinant with linear elements are completely determined.

17. Professor E. L. Dodd: The arithmetic mean of the least and greatest of $n$ measurements.

Let $P(x)$ be the probability that the error of a measurement will be algebraically less than $x$, with $P(b)=1, b$ finite or

* Journal für Mathematik, vol. 74 (1869).

$\dagger$ Proceedings of the American Academy, vol. 55, No. 7, June, 1920. 
$+\infty$; and let $P(x)$ have a continuous derivative $\phi(x)$. Let $\psi(z)$ be the probability density of the error $z$ of the arithmetic mean of the least and greatest of $n$ measurements; i.e., $\psi(z) d z$ is the probability that this error lies between $z$ and $z+d z$. Then

$\psi(z)=2 n(n-1) \int_{z}^{b}[P(x)-P(2 z-x)]^{n-2} \phi(x) \phi(2 z-x) d x$.

Suppose that the error curve $y=\phi(x)$ for the individual measurements meets the $X$-axis perpendicularly at $\pm b$, like certain Pearson curves, and that the slope of this curve is numerically greater than any given $G$ in some vicinity of $\pm b$. The curve $y=\phi(x)$, however, need not be symmetrical. Then, asymptotically for large $n$, the probability that the arithmetic mean of the least and greatest measurement will differ from the true value by less than $e$ is greater than the probability that the arithmetic mean of all the measurements will so differ. Again, if $\phi(x)=c$, a constant in $(-1 / 2 c, 1 / 2 c)$, the arithmetic mean of the least and greatest measurement is better than the arithmetic mean of all the measurements.

18. Dr. Einar Hille: Convex distribution of the zeros of SturmLiouville functions.

Dr. Hille considers the zeros of a solution of a differential equation $w^{\prime \prime}+G(z) w=0$, in a convex region $B$ in which $G(z)$ is analytic and $\vartheta_{2} \leqq \arg G(z) \leqq \vartheta_{1},\left(\vartheta_{1}-\vartheta_{2}<2 \pi\right)$. It is shown that all the zeros of $w(z) w^{\prime}(z)$ in $B$, where $w(z)$ is a solution, can be joined by a polygonal line $p_{0}$, composed of oriented line-segments, each of which forms an angle $\theta_{P}$ with the positive real axis subject to the condition $-\vartheta_{1} / 2<\theta_{P}<-\vartheta_{2} / 2$. Furthermore, let the zeros $a_{1}, a_{2}$, $\cdots, a_{n}$ be numbered in the order in which they occur on $p_{0}$. If $\arg G(z)$ is never increasing along a certain set of polygonal lines of the same type as $p_{0}$, then $\arg \left(a_{\nu+1}-a_{\nu}\right)$ is a neverdecreasing set of angles. In this case the points $\left(a_{\nu}\right)$ form the vertices of a convex polygon. Finally a zero-free region is determined for a given particular solution; the form of this region depends only on the argument of $G(z)$.

19. Professor D. R. Curtiss: On Kellogg's diophantine problem.

Professor Kellogg has proposed the problem of determining the maximum $x$ that can occur in a solution in positive integers of the equation 


$$
\frac{1}{x_{1}}+\frac{1}{x_{2}}+\cdots+\frac{1}{x_{n}}=1 .
$$

In a recent paper (American Mathematical Monthly, vol. 28, p. 300) he gives reasons for believing that the maximum $x$ is $u_{n}$, where $u_{1}=1$, and $u_{k+1}=u_{k}\left(u_{k}+1\right)$. Professor Curtiss proves this by solving the above equation for $x_{n}$ and showing that the maximum value of the resulting expression for integral values of $x_{1}, x_{2}, \cdots, x_{n-1}$ which make it positive and finite but not necessarily integral, is $u_{n}$ as defined above.

20. Dr. J. S. C. Glashan: The isodyadic quintic equation.

The object of this paper is to enable one to write down any number of isodyadic quintics independently of any knowledge of their roots. This is done by equating to zero the constant term of the diacrinic sextic given in the author's paper, On the determination and solution of the metacyclic quintic equations with rational coefficients, AMERICAN Journal, vol. 23, p. 49.

21. Dr. J. S. C. Glashan: On the isodyadic septimic equations.

The object of this paper is to determine whether any given septimic equation is isodyadic, and, if it be so, to solve it. The general discussion is followed by application to the investigation and solution of four equations with numerically assigned coefficients. Following these examples there are given the solutions of the adyadic septimics and De Moivre monodyadic septimic.

22. Professor C. F. Gummer: Criteria for relative root distributions.

In a paper presented in September, 1920, the author developed a rational method to determine the mutual arrangement of the real roots of a system of real polynomials. In the case where $g(x)$ has roots $\beta_{1}, \beta_{2}, \cdots, \beta_{q}$, all real and distinct, and arranged in descending order, it was found, under reasonable restrictions, that the number of roots of $f(x)$ between $\beta_{r}$ and $\beta_{r+1}$ could be evaluated as the coefficient of $t^{r}$ in a generating function of the form $\sum_{s=1}^{q} P_{s}(1+t)^{q-s}(1-t)^{s}$. The constants $P_{s}$ were there determined by means of the Sturmian functions of $g(x)$. In the present paper it is shown that $P_{s}$ is the signature of a certain quadratic form whose coefficients are symmetric functions of the roots of each of the polynomials $f$ and $g$. No use is made of Sturmian functions. When $g$ has $k$ pairs of imaginary roots, the same rule will determine the 
proper generating function after removal of a certain extraneous factor. A modification of this method is also given which, while depending on a less simple quadratic form, is more easily connected with the coefficients of $f$ and $g$.

\section{Professor Samuel Beatty: The algebraic theory of alge-} braic functions.

The general aim kept in view in the paper is to attain the simplicity and flexibility of treatment implied in deriving properties relative to a given basis from those relative to certain appropriate bases, the study of which presents less difficulty. Use is made of order-numbers of a certain type of adjointness relative to a given value of the variable. A lower bound is obtained for the number of linearly independent reduced forms of rational functions built on certain bases relative to a given value of the variable and containing none but negative powers of the element. Upper and lower bounds are obtained for the number of linearly independent reduced forms of rational functions built on a basis,-in the latter case, a basis of a certain type. The proof of the complementary theorem is effected by noting that were it to fail in any given case certain of the numbers obtained as lower bounds would not be such. The complementary theorem is used to obtain the number of conditions applicable to the reduced form of a rational function of a certain general type to build it on a given basis relative to a given value of the variable.

24. Professor I. R. Pounder: An algebraic proof of the existence of the branches of an algebraic function.

Under the assumption that the repeated application of Newton's process fails to separate the branches of an algebraic function $y$, subject to the relation $f(x, y)=0$, this paper shows that there exists an expression $P\left(x^{1 / \lambda}\right)+x^{t / \lambda} y^{\prime}$, where $P$ indicates a polynomial, which when substituted for $y$ in $f(x, y)$ and $f_{y}(x, y)$ makes both the resulting expressions divisible by an arbitrarily high power of $x$. This being impossible when $f(x, y)$ contains no repeated factor of the same type as itself, the validity of Newton's process is established.

25. Professor E. H. Moore: On the determinant of an hermitian matrix of quaternionic elements.

Consider a square array or matrix $K_{n} \equiv\left\|a_{f g}\right\|$ of $n^{2}$ 
quaternions $a_{f g},(f, g=1, \cdots, n)$. The matrix $K_{n}$ is hermitian in case $\bar{a}_{f g}=a_{g f}$. Here for a quaternion

$$
q \equiv c_{0}+c_{1} i+c_{2} j+c_{3} k, \quad\left(c_{0}, \cdots, c_{3} \text { real numbers }\right)
$$

$\bar{q}$ denotes the conjugate quaternion. In view of the noncommutativity of multiplication of quaternions there is probably no very useful definition of the determinant of an unconditioned quaternionic matrix $K_{n}$. However, for an hermitian quaternionic matrix $K_{n}$ a useful determinant, det. $K_{n}$, is definable as follows, by suitable determination of the order of the $n$ factors of the $n !$ terms $t$ of the formal classical det. $K_{n}$. Let the factors of a term $t$ be arranged in closed cycles $\left(f_{1} f_{2} \cdots f_{k}\right) \equiv a_{f_{1} f_{2}} a_{f_{2} f_{3}} \cdots a_{f_{k} f_{1}}$ of order $k(1 \leqq k \leqq n)$ with distinct indices $f_{1}, \cdots, f_{k}$ of $1, \cdots, n$, of which the leading index $f_{1}$ is the smallest. Let the cycles of the term $t$ be arranged according to increasing orders $k$, those of the same order $k$ being arranged according to increasing initial indices $f_{1}$. Then the quaternionic det. $K_{n}$ is the formal det. $K_{n}$ with this arrangement of the factors of each term. As an application of this definition, consider $n m$-partite quaternions or $m$-ary quaternionic vectors $\xi_{g} \equiv\left(b_{1 g}, \cdots, b_{m g}\right)(g=1, \cdots, n)$ and set $a_{f g} \equiv \bar{b}_{1 f} b_{1 g}+\cdots+\bar{b}_{m f} b_{m g}(f, g=1, \cdots, n)$. Then the matrix $K_{n} \equiv\left\|a_{f_{g}}\right\|$ is hermitian, and the vanishing of det. $K_{n}$ is a necessary and sufficient condition that the vectors $\xi_{1}, \cdots, \xi_{n}$ be linearly dependent on the right, that is, that $a_{1}, \cdots, a_{n}$ not all 0 exist such that $\xi_{1} a_{1}+\cdots+\xi_{n} a_{n}=0$.

26. Professor E. J. Wilczynski: Some properties of the surfaces which represent the real and imaginary components of a function of a complex variable.

The surfaces obtained by plotting separately the real and imaginary components, $u(x, y)$ and $v(x, y)$, of a function $w=u+i v$ of a complex variable $z=x+i y$, possess notable geometric properties which seem to have escaped notice so far. Professor Wilczynski calls attention to some of these: they are connected with the (imaginary) conjugate nets corresponding, on the $u$-surface and the $v$-surface, to the null-lines of the $x y$-plane, the (real) associate conjugate nets, and the (real) asymptotic nets of the two surfaces, where the term associate conjugate net is used in the sense of Green; i.e., at any surface point the tangents of two associate conjugate nets divide each other harmonically. The asymptotic net of the $u$-surface projects into an orthogonal isothermal net in the $x y$-plane 
which is at the same time the projection of the above-mentioned real conjugate net of the $v$-surface, and similarly for the asymptotic net of the $v$-surface. The two orthogonal nets obtained in this way in the $x y$-plane are so related that each bisects the angles determined by the other.

27. Professor O. E. Glenn: Note on differential invariants. This note relates to differential parameters for a quaternary differential form, with the condition

$$
d x_{1}{ }^{2}+d x_{2}{ }^{2}+d x_{3}{ }^{2}-d x_{4}{ }^{2}=d x_{1}{ }^{2}+d x_{2}{ }^{2}+d x_{3}{ }^{2}-d x_{4}{ }^{2},
$$

and the case where the characteristic equation determining a set of covariant differentials is a reciprocal equation.

28. Professor Louise D. Cummings: Hesse's associated points and the Weddle surface.

The theory of eight associated points from the geometric standpoint is already classic. The purpose of the present paper is to define a point by a symmetric covariant of seven other points, to establish from this equation its geometric properties, and eventually to show the point to be identical with Hesse's eighth point.

29. Professor I. J. Schwatt: Some of the principles of the operation with series applied to a partial fraction problem.

By means of some of the principles of the operation with series Professor Schwatt has obtained the partial fractions of

and

$$
\left(\sum_{k=0}^{k=n} m_{k} x^{n-k}\right) /(x+a)^{p}, n \geqq p,
$$

$$
\left(\sum_{\substack{k=n \\ k=0}}^{k} m_{k} x^{n-k}\right) /\left(x^{2}+a x+b\right)^{p}, n \geqq 2 p .
$$

The coefficients of the first are expressed as single summations and those of the second as double summations. The literature on the subject shows that heretofore the coefficients have been expressed as determinants.

30. Professor I. J. Schwatt: Expansion of powers of infinite series.

If the series to be expanded to the $p$ th power is not summable, the coefficient of the general term of the expansion is the product of $p-1$ summations. If the series is summable the work is reduced to the evaluation of the general derivative 
of a function of a function for the vanishing primary variable. The methods employed are believed to be new.

31. Professor Olive C. Hazlett: A symbolic theory of formal modular invariants.

At the meeting in connection with the Chicago Colloquium, Professor Hazlett introduced a symbolic notation for the formal modular invariants of a binary form $f$ of degree $m$ with respect to the general Galois field $G F\left[p^{n}\right]$ of order $p^{n}$. To this end, the form $f$ was expressed as the product of $m$ symbolically distinct factors $\alpha_{x}=\alpha_{1} x_{1}+\alpha_{2} x_{2}, \beta_{x}=\beta_{1} x_{1}+\beta_{2} x_{2}, \ldots$. It was there shown that all formal modular invariants of $f$ are expressible as polynomials in a finite number of symbolic expressions which behave like invariants. These invariant symbols include determinantal symbols of the type

$$
(\alpha \beta)=\alpha_{1} \beta_{2}-\alpha_{2} \beta_{1},
$$

which arise in the theory of ordinary invariants of $f$, and more complicated ones which are peculiar to the theory of modular invariants. In the present paper, it is shown that, if a formal modular invariant $I$ is isobaric, it is necessarily congruent (modulo $p$ ) to a polynomial in determinantal symbols of type $(\alpha \beta)$ and hence is an ordinary invariant of $f$, though not necessarily a rational one. In case $I$ is not isobaric, then it is congruent (modulo $p$ ) to an ordinary invariant (rational or irrational) of $f$ and certain related forms. Similar theorems hold for formal modular covariants of $f$.

32. Dr. Norbert Wiener and Dr. J. L. Walsh: The equivalence of expansions in orthogonal functions.

The authors develop necessary and sufficient conditions for the identity of the convergence properties of different expansions in orthogonal functions. They apply these notions to various related problems. The chief theorem is that two closed sets of normal orthogonal functions $\left\{\varphi_{n}\right\}$ and $\left\{\psi_{n}\right\}$ have identical convergence properties when and only when the $\varphi$-series for the $\psi$ 's converge uniformly, while

$$
\int_{0}^{1}\left\{\sum_{1}^{n}\left[\varphi_{n}(x) \varphi_{n}(y)-\psi_{n}(x) \psi_{n}(y)\right]\right\}^{2} d y
$$

is uniformly bounded in $n$ and $x$.
Arnold Dresden,
R. G. D. RIChaRdSON, Secretary of Chicago Section.
Secretary. 